\title{
BIBLIOGRAFIA
}

\section{Rómulo Gallegos: Suplemento a una Bibliografía}

El reciente libro de Efraín Subero: Contribución a la bibliografía de Rómulo Gallegos, Caracas, Universidad Católica Andrés Bello, 1969, 405 p. constituye una valiosa aportación a los estudios sobre el gran novelista venezolano. La importancia de Gallegos exige que una bibliografía semejante sea lo más completa posible. Por este motivo hemos reunido en la presente lista una serie de referencias a libros, folletos y artículos, los cuales, por razones que todo bibliógrafo fácilmente comprenderá, faltan en la obra de Subero. Hemos incluido referencias a varios libros y artículos publicados después de 1969 que el mismo profesor Subero amablemente nos ha facilitado. Prescindimos de una larga lista de tesis y de artículos en periódicos menores que Lowell Dunham incluyó en su conocido libro Rómulo Gallegos - vida y obrä, México, Andrea, 1957.

Donald L. SHAW

University of Edinburgh

\section{Suplemento Bibliográfico}

Agudo Freites, R., "Doña Bárbara y el latifundio", Contrapunto (Caracas); II (abril-mayo de 1948), pp. 19-22.

"L'Aiglon", "Notas teatrales", La Revista (Caracas), Serie IV, 22 y 29 de agosto de 1915; Serie V, 5, 12 y 19 de septiembre de 1915; Serie VII, 6 de noviembre de 1915. 
Alegría, Fernando, "Rómulo Gallegos", Revista Iberoamericana, XXXVI, No 70 (enero-marzo de 1970), pp. 61-63.

Allen, R. F., "Consideraciones sobre aspectos socio-políticos de la obra de Rómulo Gallegos", Duquesne Hispanic Review, IV, No I (primavera de 1965), pp. 25-38.

Anónimo, "Rómulo Gallegos", Sur, No 232 (enero-febrero de 1955), pp. 63-64.

Anónimo, "Search for a Country's Soul", Times Literary Supplement 10 de febrero de 1961, p. 88.

Anónimo, "Rómulo Gallegos cumple 80 años de impulso creador", $E l$ Libro y el Pueblo (México), IV, No 16, 1964, pp. 16-17.

Anónimo, "Entrevista con Rómulo Gallegos", Excelsior (México), 14 de octubre de 1965.

Anónimo, Vida y obra de Rómulo Gallegos, Madrid, Edit. Siglo Ilustrado, Col. La Tijera Literaria, No 38, 1970.

Añez, J., De La vorágine a Doña Bárbara, Bogotá: Imprenta del Departamento, 1944, $213 \mathrm{pp}$.

Araujo, Orlando, "El folklore en Rómulo Gallegos", Arcbivo Venezolano de Folklore (Caracas), I, No 2, 1952, pp. 323-337.

Araujo, Orlando, "La obra de Rómulo Gallegos", Insula, No 272/3 (julio-agosto de 1969), p. 25.

Arciniegas, Germán, "Venezuela's Novelist President", United Nations World (New York), II, No 3 (abril de 1948), pp. 60-61.

Arciniegas, Germán, "El viejo Gallegos", El Nacional (Caracas), 4 de junio de 1967 , p. 4.

Arévalo Martínez, Rafael, "Novelas Americanas I, Doña Bárbara", Boletin de la Biblioteca Nacional (Guatemala), I, No 3, (noviembre de 1932), pp. 84-85.

Armas Alfonzo, Alfredo, "I. p.m. la hora del general Guaviare", Imagen (Caracas), No 70/71 (abril de 1970), pp. 3-4.

Asturias, Miguel Angel, "Originalità e caratteristiche del romanzo latino-americano", Terzo Programa (Roma), IV, 1964, pp. 51-74.

Aubrun, Charles, "Langue et style, à propos d'une étude sur Rómulo Gallegos", Les Langues Neo-Latines (Paris) 144, 1958, pp. 49-52.

Azuela, Salvador, "Homenaje a Rómulo Gallegos", El Libro y el Puebla (México) No 7/8 (julio-agosto de 1954), pp. 65-68.

Barbagelata, Hugo, La novela y el cuento en Hispanoamérica, Montevideo, Enrique Míguez, 1947, 316 pp.

Bellini, Giuseppe, "La narrativa de Rómulo Gallegos", America Latina (Milán), I, No. 1, 1952, p. $60 f f$. 
Bellini, Giuseppe, La protesta nel romanzo ispano-americano del 900 , Milán-Varese, Cisalpino, 1957, $80 \mathrm{p}$.

Bellini, Giuseppe, Il romanzo de Rómulo Gallegos, Milán, Goliardica, 1962, $64 \mathrm{pp}$.

Berenguer Carisomo, Arturo y Bogliano, Jorge, Medio siglo de literdtura americana, Madrid, Cultura Hispánica, pp. 176-237.

Betancourt, Rómulo, "Apuntes para una interpretación de Doña Bárbara, Repertorio Americano (San José, Costa Rica), Año 12, No 509 (4 de octubre de 1930), pp. 201-202.

Blanco, Andrés Eloy, "Doña Bárbara, de lo pintado a lo vivo", Humanismo (México), I, № 6 (diciembre de 1952) pp. 18-19; también La mañana (Montevideo), 8 de marzo de 1953.

Bollo, Sarah, "Páginas críticas II: La novela de Rómulo Gallegos", Revista Nacional (Montevideo), XLII, No 125 (mayo de 1949) pp. 186-199.

Bosch, J., "De Don Quijote a Doña Bárbara", Humanismo (México), III, No 22 (agosto de 1954), pp. 31-35.

Bueno, Salvador, "La novela cubana de Rómulo Gallegos", Carteles (La Habana), XXXIII, No 13 (30 de marzo de 1952), pp. 50-52.

Bueno, Salvador, "Don Rómulo y Doña Bárbara", Revista de la Bibliateca Nacional (La Habana), VI, No 2, 1955, 233-237.

Camp. J., "Um grande romancista: Rómulo Gallegos", Cadernos Brasileiros II, No 4, 1960, pp. 48-52.

Campos, Jorge, "Rómulo Gallegos", Insula, № 270 (mayo de 1969), pp. 10-11.

Cárdenas, Lázaro, "De Lázaro Cárdenas a Rómulo", Novedades (México), No 292 (24 de octubre de 1954), pp. 1-2.

Carrión, Benjamín, "Rómulo Gallegos y John Dos Passos", Novedades (México) No 42 (2 de diciembre de 1956), pp. 4-6.

Carrión, Jorge, "El descendiente del Cunavichero", Humanismo (México), III, No 22 (agosto de 1954), pp. 109-111.

Carruther, Ben, F., "Gallegos: Doña Bárbara The Llanos Personified", The Panamerican (Nueva York), X, No 8 (10 de enero de 1950), pp. 19-20.

Castanien, Donald, G., "Introspective Techniques in Doña Bárbara" His. pania (AATSP), XLI, No 3 (septiembre de 1958), pp. 282-288.

César, Armádio, "Rómulo Gallegos na literatura sul-americana", Tempo Presente (Lisboa), III, No 27, 1961, pp. 60-64.

Coester, Alfred, "Maelstroms, Green Hells and Sentimental Jungles", Hispania (AATSP), XVI, No 1 (marzo de 1933) pp. 33-50. 
Coronel, Rafael, "Canaima, novela por Rómulo Gallegos", Anales de la Universidad de Chile, 3ra. Serie, XCIV, No. 4 (octubre de 1936), pp. 293-295.

Correa, Gustavo, "El mundo metafórico de Doña Bárbara" en Actas del Sexto Congreso del Instituto Nacional de Literatura Iberoamericana, México, Imprenta Universitaria, 1954, pp. 227-233.

Correa, Gustavo, "Aspectos del tema de la selva en Rómulo Gallegos y Horacio Quiroga", Revista Nacional de Cultura (Caracas), No 127 (marzo-abril de 1958), pp. 12-22.

Crema, Eduardo, "Características diferenciales de Doña Bárbara", Imagen (Caracas), No 70/71 (abril de 1970), pp. 5-8.

Crespo de la Serna, José, "El pintor en Daña Bárbara", Humanismo (México), III, N 26 (diciembre de 1954), pp. 60-68.

Crow, John, A., "A Critical Appraisal of the Contemporary SpanishAmerican Novel", Hispania (AATSP), XXIV, No 2 (mayo de 1951), pp. 155-164.

Crow, John, A., "Perspectiva de la novela en Rómulo Gallegos", $H u$ manismo (México), III, No 22 (agosto de 1954), pp. 37-40.

Chapman, A., "The Barefoot Galateas of Bret Harte and Rómulo Gallegos", Symposium (Syracuse), XVIII, No 4 (invierno de 1964), pp. 332-341.

Dumont, Maurice, Alex, "Rómulo Gallegos et les centaures du Venezuela", La Revue Nowvelle (Bruselas), XVIII, No 9 (15 de septiembre de 1953), pp. 182-189.

Dunham, Lowell, Rómulo Gallegos: A Biograpbical Study, Universidad de California, Los Angeles, 1956, 465 pp. (Tesis).

Dunham, Lowell, "Rómulo Gallegos creador de la literatura nacional venezolana", Revista Nacional de Cultura (Caracas), No 164 (mayojunio de 1964), pp. 32-38.

Dunham, Lowell, "Erudito, novelista y presidente: el venezolano Rómulo Gallegos", Las Américas (Washington), XVII, No 1 (enero de 1965), p. 1-5.-También Vida Universitaria (Monterrey) 11 de abril de 1965, pp. 3-11.

Dunham, Lowell, "Rómulo Gallegos 1884-1969", Books Abroad (Oklahoma), No 43 (verano de 1969), pp. 368-369.

Englekirk, John E., "Algunas fuentes de Doña Bárbara", Número (Montevideo), III, No:13/14, 1951, pp. 204-209.

Fabbiani Ruiz, José, "Novela y paisajismo", El Nacional (Caracas), 5 de agosto de 1957 , p. 4. 
Fitżgerald, T. A., "Notas sobre Doña Bárbara", Hispania (AATSP), XXVI, No I (enero de 1943), pp. 117-118.

Franulic, Lenka, "Rómulo Gallegos" en Cien autones contemporáneos, Tercera Ed. Santiago de Chile, Ercilla, 1952, pp. 293-301.

Galaos, José Antonio, "Rómulo Gallegos o el duelo entre civilización y barbarie", Cuadernos Hispanodmericanos, LV, No 165, 1963, pp. 299-309.

Gallegos, Rómulo, La brasa en el pico del cuervo (los dos primeros ca. pítulos) Cuadernos Americanos, CLXIV, 3, (mayo/junio de 1969) pp. 163-201.

García Valencia, Abel, "Notas y comentarios: El forastero", Universidad de Antioquia (Medellín), No 55 (octubre-noviembre de 1942), pp. 282-283.

Garmendia, Salvador, La novela en Venezueld, Caracas, Oficina Central de Información, 1967, 24 pp.

González, Manuel Pedro, "Del momento hispanoamericano -a propósito de Doña Bärbara", Bulletin Of Spanish Studies (Liverpool), VII, No 28 (octubre de 1930), pp. 162-167.

González, Manuel Pedro, "Sobre Rómulo Gallegos, Cantaclaro y Candsma", Revista Cubana (Santiago), III, No 121-127, 1935, p. 6ff.

González, Manuel Pedro, "Apostillas a la última novela de Rómulo Grllegos", Humanismo (México), III, № 22 (agosto de 1954), pp. 71-76.

González, Manuel Pedro, "Crisis de la novela en América", Revisła Nacional de Cultura (Caracas), № 150 (enero-febrero de 1962), pp. 50-69. También en Ensayos criticos, Caracas, Universidad Central, 1967 y en Coloquio sobre la novela bispanoamericand, México, Tezontle, 1967, pp. 37-67.

Guillén, Fedro, "Rómulo Gallegos, realidad y símbolo", El Nacional (Caracas), 3 de octubre de 1967.

Hutton, E. R., "Two Venezuelan Books", Hispania (AATSP), XXIX, № 1 (enero de 1946), pp. 84-87.

Iduarte, Andrés, "Rómulo Gallegos, novelista de América", Universidad Obrera de México, No 7 (abril de 1936), pp. 1-18.

Iduarte, Andrés, "Carta a Rómulo Gallegos", Cuadernos Americanos, VIII, No 1 (enero-febrero de 1949), pp. 8-15.

Iduarte, Andrés, "Con Rómulo Gallegos en España", Revisía Hispánica Moderna, XXX, No 1 (enero-octubre de 1965), pp. 230-235.

Jackson, Richard L., "Miscegenation and Personal Choice in two Twen- 
tieth Century Novels of Continental Spanish America", Hispania (AATSP), L, No 1 (marzo de 1967), pp. 86-88.

Johnson, E. A., "The meaning of civilization and barbarie in Doñt Bárbara", Hispania (AATSP), XXXIX, No 4 (diciembre de 1956). pp. 456-461.

Jones, Cyril A., Three Spanish American Novelists, London, Hispanic and Luso-Brasilian Councils, Diamante Series, No 17, 1967, 24 p.

Kirsner, R., "Una nota sobre la novela de Rómulo Gallegos", en Actas del Sexto Congreso del Instituto Internacional de Literatura Ibero. americana, México, Imprenta Universitaria, 1954, pp. 223-226.

Kolb, Glen L., "Aspectos estructurales de Doña Bärbard", Revista lberoamericana, XXVIII, No 53 (enero-junio de 1962), 131-140.

Kolb, Glen L., "Dos novelas y un solo argumento", Hispania (AATSP), XLVI, N 1 (marzo de 1963), 84-87.

Krebs, Ernesto, Marianela y Doña Bárbara: ensayo de comparación, Bahía Blanca, Universidad Nacional del Sur, 1967, 177 pp.

Lacosta, Francisco C., "Notas sobre una novela de la tierra: Rómulo Gallegos, Cantaclaro", Romance Notes, V, No 2 (diciembre de 1963). pp. 107-111.

Landa, Rubén, "La personalidad moral de Gallegos", Cuadernos Americanos, XX, No 1 (enero-febrero de 1960), pp. 61-94.

Leavitt, Sturgess E., "Sex versus symbolism in Doña Bârbara", Revista de Estudios Hispánicos (Alabama) I, No 1 (mayo de 1967), pp. 117-120.

Leo, Ulrich, "Sobre la misma tierra", Revista Nacional de Cultupa (Caracas) No 52 (julio-agosto de 1945), pp. 55-74 y No 55 (septiembre-octubre de 1945), pp. 75-91.

Leo, Ulrich, "Un maestro en formación", Revista Hispánica Moderns. IX, No $1 / 2$ (enero-abril de 1945), pp. 19-36.

Leo, Ulrich, "Rómulo Gallegos", Romanische Forscbungen, LXVI, No 3/4 (diciembre de 1955), pp. 239-254.

Liscano, Juan, Ciclo y constantes galleguianos, México, Ediciones Humanismo, 1954, 45 p., también en Imagen (Caracas), No 70/71 (abril de 1970), pp. 14-23.

Liscano, Juan, "Rómulo Gallegos", Las Américas (Washington), VII, No 11 (noviembre de 1954), p. 4.

Liscano, Juan, "Rómulo Gallegos y su obra", Revista de la Universidad de Zulid (Venezuela), 2da. Epoca, I, No 4, 1958, pp. 103-118.

Liscano," Juan, "Encuentro con Rómulo Gallegos", Cuademos (París) No 6 (mayo-junio de 1954), pp. 17-24; también en Expresión del peinsamiento contemporáneo, Buenos Aires, Sur, 1965, pp. 303-317. 
Liscano, Juan, "La obra literaria de Rómulo Gallegos", Revista Interamericana de Bibliografia XVI, No 2 (marzo de 1966), pp. 144 151.

Liscano, Juan, "Rómulo Gallegos", Vida Universitaria (Monterrey), 19 de marzo de 1967, pp. 12-15.

Liscano, Juan, La geografía venezolana en la obra de Rómulo Gallegor, Caracas, Ministerio de Educación de Publicaciones, 1970, 133 p.

Loreley (seudónimo), "Rómulo Gallegos, el escritor y el hombre", Repertorio Americano (San José, Costa Rica), Año XVIII, No 805 (5 de junio de 1937), pp. 329-331.

Loveluck, Juan, "Los 25 años de Doña Bárbara", Atenea (Santiago de Chile), XXXII, N2 359, 1954, pp. 153-174.

Llerena, M., "Función del paisaje en la novela hispanoamericana", Hispania (AATSP), XXXII, No 4 (diciembre de 1949), pp. 499-503.

Magdaleno, Mauricio, "Imágenes políticas de Rómulo Gallegos", Cuadernos Americanos, X, No 6 (noviembre-diciembre de 1951), pp. 234-239.

Maestri, José A., "Doña Bárbara", Humanismo (México), III, No 23 (septiembre de 1954), pp. 77-79.

Mañach, Jorge, "Una gran novela americana", Humanismo (México), III, No 22 (agosto de 1954), pp. 25-29.

Marinello, Juan, "Tres novelas ejemplares", Sur, № 16 (enero de 1936, 59-75); también Revista Bimestre Cubana, XXXVIII, No 2 (septiembre-diciembre de 1936), pp. 234-249.

Mata, Ramiro, W., Güiraldes, Rivera, Gallegos: estudios biocríticos, Montevideo, CISA, 1961, 224 p.

Mata Requezas, Luis, "Doña Bárbatra y La vorágine", Revista del Instituto Pedagógico Nacional (Caracas), febrero-marzo de 1946, 85-87.

Medina, José Ramón, Rómulo Gallegos, ensayo biográfico, Caracas, Arte, 1966, 86 p.

Meléndez, Concha, "Tres novelas de la naturaleza americana", Culturs Venezolana (Caracas), XIII, No 107 (noviembre de 1930), PP 138-149.

Meléndez, Concha, "Dan Segundo Sombrd, La vorágine, Doña Bárbara", Cultura Venezolana (Caracas), XIV, No 116 (noviembre de 1931), pp. 121-132.

Meléndez, Concha, "Sobre Rómulo Gallegos, Doña Bárbara", en Signos de Iberoamérica, México, Manuel L. Sánchez, 1936, pp. 91-100.

Meléndez, Concha, "El mito de los ríos en dos novelas hispanoamericanas", Viernes (Caracas), I, No 8 (marzo de 1940), pp. 26-29. 
Menéndez, S., "Rómulo Gallegos prepara su próximo libro, El forastero", Debate (Madrid), 26 de septiembre de 1931, p. 4.

Michalski, S., "Doña Bárbara, un cuento de hadas", Publications of the Modern Language Society of America, LXXXV (octubre de 1970) pp. 1015-1022.

Molinaro, J., "Doña Bárbara y Pigmalión", Quademi Iberoamericani (Torino), III, 1956, pp. 212-215.

Montañes, Domingo, "Rómulo Gallegos", Horizontes (México), VII, No. 39, 1964, pp. 6-8.

Montenegro, E., "The Literary Scene in South America", New York Times Book Review, 30 de marzo de 1941, pp. 8 y 27.

Montilla, Ricardo, "Rómulo Gallegos", Novedades (México), No 292 (24 de octubre de 1954), pp. 2-6

Morales, Angel Luis, "El naturalismo en los cuentos de Rómulo Gallegos", Duquesne Hispanic Review, V, No 1 (primavera de 1962), pp. 24-40.

Morales, Angel Luis, La naturaleza venezolana en la obra de Rómulo Gallegos", Puerto Rico, Editorial del Departamento de Instrucción Pública, 1969, $378 \mathrm{p}$.

Mörner, Magnus, La voz de Venezuela en el concierto de la literatura muntial: Rómulo Gallegos, Biblioteca de Estudios Iberoamericanos de la Escuela de Ciencias Económicas, Estocolmo, 1960, 16 p.

Moton Guillermo, "Introducción a unas lecciones universitarias" El Nacional (Caracas), 7 de marzo de 1957, p. 6.

Núñez y Domínguez, José de Jesús, La novela contemporánea de Hispanartmérica y Rómulo Gallegos, México, Departamento del D. F., Dirección de Acción Social, 1944, 7 p.

Okinshevich, Leo y Carlton, Robert G., Latin America in Soviet Writings, Baltimore, John Hopkins, 1966, 2tt. 257 y 311 p.

Ordóñez Argüello, Alberto, "Gallegos en Guatemala", Nuestra Guatemala V, No 12 (marzo de 1951), pp. 15-16 y 46.

Osorio, Luis E., "Rómulo Gallegos" en Democracia en Venezuela, Bogotá, 1943.

Owre, J. Riis, "The fauna of the works of Rómulo Gallegos", Hispania (AATSP), XLV, No 1 (marzo de 1962), pp. 52-56.

Palacios, Lucila, "Una interpretación de la novela de Rómulo Gallegos", Revista Nacional de Cultura (Caracas), No 164 (mayo-junio de 1964), pp. 21-31.

Palacios, Lucila, "Bienvenida a Rómulo Gallegos", El Universal (Caracas) 6 de noviembre de 1966, p. 4. 
Pardo Tovar, R. A., "Rómulo Gallegos, novelista de América", Revista de Indias (Bogotá), II, 66/67 (junio-julio de 1944), pp. 165-188.

Paz Castillo, Fernando et al., "Fragmentos de algunos juicios críticos que mereció la obra de Rómulo Gallegos Doña Bárbara, addendum a La trepadora, 2da. ed., Barcelona, Araluce, 1930, 3 p.

Peña, Margarita, "El diablo, aliado y socio en Doña Bárbara", Mundo Nuevo (París), 48, (junio de 1970) pp. 65-69.

Peralta, Jaime, "Una aproximación a la idea de América en Rómulo Gallegos", Cuadernos Hispanoamenicanos, LX, No 178, 1964, pp. 136-145.

Pérez, Galo René, "Cantaclaro, llanero de la copla errante", Letras del Ecuador (Quito), XX, No 130, 1964, pp. 3 y 18.

Pietsch, Franz, "Rómulo Gallegos, Doña Bárbara", Die Nueren Sprachen, VIII, No 5, 1959, 236-242.

Pineda, Rafael, "Cantaclaro, contrapunteo de fantasmas", Imagen (Caracas), No 70/71 (abril de 1970), pp. 38-40.

Piña-Daza, R., "Un curioso personaje de nuestra novelística", Revist.t Nacional de Cultura (Caracas), No 41 (noviembre-diciembre de 1943), pp. 33-44.

Portuondo, José Antonio, "Rómulo Gallegos, Pobre Negro", AfroAmérica (México), II, No 3, 1946, pp. 155-156.

Prieto Figueroa, Luis B., "Puntos de vista, Rómulo Gallegos", Atenea, XXV, No 274 (marzo de 1948), pp. 165-168.

Ramos, Jorge, "Rómulo Gallegos visto por un escritor portugués", Horizontes (México), IX, No 47, 1966, p. 15.

Rangel Guerra, Alfonso, "La ruta de los libros, Doña Bárbara: novela americana", Vida Universitaria (Monterrey), V, No 195, 1954, 16-23.

Ratcliffe, Dillwyn Fritschell, "Sobre Rómulo Gallegos, Doña Bärbara", Cultura Venezolana (Caracas), XII, No 101, 1929, 146-149.

Ratcliffe, Dillwyin Fritschell, "Three Recent Venezuelan Novels", Hispania (AATSP), XX, No 1 (marzo de 1937), 27-30.

Reid, John T., "Spanish American Jungle Fiction", Quarterly Journal of Inter-American Relations (Washington), II, No 1 (enero de 1940), pp. 48-58.

Reid, John T., "Notes and Meditations on Contemporary Venezuelan Literature", Hispania (AATSP), XXXIV, № 3 (agosto de 1951), pp. 227-232.

Reyes, Alfonso, Rómulo Gallegos en Morelia, México, Publicaciones del Gobieno del Estado, 1952, 9 p.

Rodríguez Rodríguez, Adolfo, Oriente en la abra de Rómulo Gallegos, Caracas, Ministerio de Educación Publicaciones, 1970, 142 p. 
Ross, Waldo, "Meditación sobre el mundo de Juan Solito", Revista Nat cional de Cultura (Caracas), No 156-157 (enero-abril de 1963) pp. $57-72$.

Sabat Ercasty, Carlos, "La lección de Gallegos", Cuadernos Americanos, XIII, No 6 (noviembre-diciembre de 1954), pp. 77-84.

Sánchez Trincado, José Luis, "Reinaldo Solar, caballero sin espada", Viernes (Caracas), I, No 10 (mayo de 1940), pp. 14-18; también en Stendbal y otras figuras, Buenos Aires, Imprenta Cópec, 1943, $125 \mathrm{p}$.

Scarpa, Roque Esteban, Lecturas americanas, Santiago de Chile, ZigZag, 1944, 636 p.

Scheines, Gregorio, Novelas rebeldes de América, Buenos Aires, Américalee, $1960,123 \mathrm{p}$.

Schiro, Roberto, "Canaima ¿Vargas involuciona?", Universidad, Santa Fe, LXX, 1970, 149-158.

Schultz de Mantovani, Fryda, "Doña Bárbara y la América de Rómulo Gallegos", Sur, No 230 (septiembre-octubre de 1954), pp. 79-96.

Selva, M. de la, "Alrededor de Rómulo Gallegos (estudio y entrevis. ta)", Cuadernos Americanos, XV, No 5 (septiembre-octubre de 1956), pp. 256-269.

Sisto, D. T., "Doña Perfecla and Doña Bárbara", Hispania (AATSP), XXXVII, No 2 (mayo de 1954), pp. 167-170.

Sisto, D. T., "The String in the Conjurations of $\mathrm{La}$ Celestina and Doña Bárbara", Romance Nates, I, No 1 (noviembre de 1939), pp. 50-52.

Spell, Jefferson R., Contemporary Spanish American Fiction 1914-1942, Chapel Hill, University of N. Carolina, 1944, pp. 205-238.

Suárez Calimano, E., "Sobre Rómulo Gallegos, Doña Bárbard", Nosotror, XXIV, No 254-255 (julio-agosto de 1930), pp. 128-138.

Suárez Solís, R., "Gallegos y su América", Humanismo, (México),- III, No 22 (agosto de 1954), pp. 65-68.

Torre, Manuel, "Rómulo Gallegos, el rápsoda de la llanura", Repertorio Americano (San José, Costa Rica), XL, No 15 (21 de agosto de 1943), pp. $225-6$.

Torres-Rioseco, Arturo, Nouelistas contemporáneos de América, Santiago de Chile, Nascimento, 1939, pp. 90-122.

Torres-Rioseco, Arturo, La novela en la América Hispana, Berkeley, Universidad de California (Publications in Modern Philology, No 21), 1939, pp. 159-255.

Torres-Rioseco, Arturo, "Rómulo Gallegos", Atenea (Santiago de Chile), XVI, No 169, 1939, pp. 81-112. 
Torres-Rioseco, Arturo, "Tres aspectos de la personalidad de Rómulo Gallegos", Elite (Caracas), 13 de septiembre de 1947, pp. 3-4.

Trillas, Gabriel, "Conózcalos en pocas palabras: Rómulo Gallegos", $R_{t}$. vista de América (Bogotá), XIII, No 37 (junio de 1948), pp. 87-89.

Urdueta, T., "Cantaclaro, el nuevo libro de Rómulo Gallegos", Atenea (Santiago de Chile), XI, № 109, 1934, pp. 62-68.

Valbuena Briones, A., " $\mathrm{La}$ idea política en la novela de Gallegos", Atenea (Santiago de Chile), XLI, No 403, 1964, pp. 84-93.

Valbuena Briones, A., Ideas y palabras, Nueva York, Las Américas Publishing Co., 1968, 125 p.

Venegas Filardo, Pascual, Novelas y novelistas de Venezuela, Caracas, Tipografía de la Nación, 1955, 57 p.

Verde, Genaro, "La obra de Rómulo Gallegos", Unión Universitaria (Universidad de Cauca, Popayán), 12 de julio de 1946, pp. 4-6.

Vian, Cesco, "Rómulo Gallegos e il romanzo latinoamericano di oggi", Vita e Pensiera (Milán), No 33, 1950, pp. 270-274.

Wais, Kurt K. T., Zwei Dicbter Südamerikas: Gabriela Mistral, Rómulo Gallegos, Berlin, H. Luchterhand, 1955, 87 p.

Welsh, Louise, "The Emergence of Rómulo Gallegos as Novelist and Social Critic", Hispania (AATSP), XL, No 4 (diciembre de 1957), pp. 444-449.

Wolfe, Bertram, D., "The Novel in Latin-America", The Antioch Review, III, No 2 (verano de 1943), pp. 191-208.

Zérega Fombona, A., "Para los ochenta años de Rómulo Gallegos", El Universal (Caracas), 8 de septiembre de 1964, p. 1. 
